\title{
GENERIC RIEMANNIAN MAPS
}

\begin{abstract}
B. ŞAHIN
Received 16 June, 2016

Abstract. As a generalization of semi-invariant Riemannian maps from almost Hermitian manifols, we first introduce generic Riemannian maps from almost Hermitian manifolds to Riemannian manifolds, give examples, obtain decomposition theorems and investigate harmonicity and totally geodesicity of such maps. Then as a generalization of semi-invariant Riemannian maps to almost Hermitian manifolds, we introduce generic Riemannian maps from Riemannian manifolds to almost Hermitian manifolds, give examples and find necessary and sufficient conditions for such maps to be totally geodesic. The harmonicity of generic Riemannian maps to Kähler manifolds is also investigated.
\end{abstract}

2010 Mathematics Subject Classification: 53C15; 58C25; 53C43

Keywords: Kähler manifold, Riemannian maps, anti-invariant Riemannian submersion, semiinvariant submersion, semi-invariant Riemannian map, generic Riemannian map, harmonic map

\section{INTRODUCTION}

As indicated in [9], a major flaw in Riemannian geometry (as compared to other subjects) is a shortage of suitable types of maps between Riemannian manifolds that will compare their geometric properties. In this direction, Fischer introduced Riemannian maps between Riemannian manifolds in [8] as a generalization of the notions of isometric immersions and Riemannian submersions. Isometric immersions and Riemannian submersions have been widely studied in differential geometry (see for examples [6] and [7]), however the theory of Riemannian maps is a new research field. Let $F:\left(M_{1}, g_{1}\right) \longrightarrow\left(M_{2}, g_{2}\right)$ be a smooth map between Riemannian manifolds. Then we denote the kernel space of $F_{*}$ by $k e r F_{*}$ and consider the orthogonal complementary space $\left(k e r F_{*}\right)^{\perp}$ to $k e r F_{*}$. Then the tangent bundle of $M_{1}$ has the following decomposition

$$
T M_{1}=k e r F_{*} \oplus\left(k e r F_{*}\right)^{\perp} .
$$

We denote the range of $F_{*}$ by $r a n g e F_{*}$ and consider the orthogonal complementary space $\left(\text { range } F_{*}\right)^{\perp}$ to range $_{*}$ in the tangent bundle $T M_{2}$ of $M_{2}$. Thus the tangent bundle $T M_{2}$ of $M_{2}$ has the following decomposition

$$
T M_{2}=\left(\text { range }_{*}\right) \oplus\left(\operatorname{range} F_{*}\right)^{\perp} .
$$

(C) 2017 Miskolc University Press 
Now, a smooth map $F:\left(M_{1}^{m}, g_{1}\right) \longrightarrow\left(M_{2}^{n}, g_{2}\right)$ is called Riemannian map at $p_{1} \in$ $M_{1}$ if the horizontal restriction $F_{* p_{1}}^{h}:\left(k e r F_{* p_{1}}\right)^{\perp} \longrightarrow\left(\right.$ range $\left._{* p_{1}}\right)$ is a linear isometry between the inner product spaces $\left.\left(\left(k e r F_{* p_{1}}\right)^{\perp},\left.g_{1}\left(p_{1}\right)\right|_{\left(k e r F_{* p_{1}}\right.}\right)^{\perp}\right)$ and $\left(\right.$ range $\left._{* p_{1}},\left.g_{2}\left(p_{2}\right)\right|_{\left(\text {range }_{* p_{1}}\right)}\right), p_{2}=F\left(p_{1}\right)$. It follows that isometric immersions are particular Riemannian maps with $\operatorname{ker}_{*}=\{0\}$. It also follows that Riemannian submersions are particular Riemannian maps with $\left(\mathrm{rangeF}_{*}\right)^{\perp}=\{0\}$. If rankF $<\min \{m, n\}$, we always have $\left(\operatorname{range}_{*}\right)^{\perp} \neq\{0\}$, in this case, we say that $F$ is a proper Riemannian map. It is known that a Riemannian map is a subimmersion which implies that the rank of the linear map $F_{* p}: T_{p} M_{1} \longrightarrow T_{F(p)} M_{2}$ is constant for $p$ in each connected component of $M_{1}$, [1] and [8]. It is also important to note that Riemannian maps satisfy the eikonal equation which is a bridge between geometric optics and physical optics. For Riemannian maps and their applications in spacetime geometry, see: [9].

In [19], we introduced semi-invariant Riemannian maps from almost Hermitian manifolds onto Riemannian manifolds as a generalization of holomorphic Riemannian maps and anti-invariant Riemannian maps, then we studied the geometry of such maps. We also introduced and studied semi-invariant Riemannian maps to almost Hermitian manifolds in [18]. Recently, there are many research papers on the geometry of Riemannian maps between various Riemannian manifolds [10], [11], [14], [15], [16].

In this paper, we introduce and study both generic Riemannian maps from almost Hermitian manifolds and generic Riemannian maps to almost Hermitian manifolds.

The paper is organized as follows: In Section 2, we present the basic background needed for this paper. In section 3, we define generic Riemannian maps from almost Hermitian manifolds to Riemannian manifolds, give examples and obtain integrability conditions for distributions defined by generic Riemannian maps. We also find necessary and sufficient conditions for a semi-invariant map to be totally geodesic map and harmonic map. In the last section of this paper, we study generic Riemannian maps to almost Hermitian manifolds and obtain main properties of such maps.

\section{PRELiminaries}

In this section we recall some basic materials from [3] and [24]. A $2 k$-dimensional Riemannian manifold $(\bar{M}, \bar{g}, \bar{J})$ is called an almost Hermitian manifold if there exists a tensor filed $\bar{J}$ of type $(1,1)$ on $\bar{M}$ such that $\bar{J}^{2}=-I$ and

$$
\bar{g}(X, Y)=\bar{g}(\bar{J} X, \bar{J} Y), \forall X, Y \in \Gamma(T \bar{M}),
$$

where $I$ denotes the identity transformation of $T_{p} \bar{M}$. Consider an almost Hermitian manifold $(\bar{M}, \bar{J}, \bar{g})$ and denote by $\bar{\nabla}$ the Levi-Civita connection on $\bar{M}$ with respect to $\bar{g}$. Then $\bar{M}$ is called a Kähler manifold[24] if $\bar{J}$ is parallel with respect to $\bar{\nabla}$, i.e,

$$
\left(\bar{\nabla}_{X} \bar{J}\right) Y=0
$$


for $X, Y \in \Gamma(T \bar{M})$.

Let $\left(M, g_{M}\right)$ and $\left(N, g_{N}\right)$ be Riemannian manifolds and suppose that $F: M \longrightarrow$ $N$ is a smooth map between them. Then the differential $F_{*}$ of $F$ can be viewed a section of the bundle $\operatorname{Hom}\left(T M, F^{-1} T N\right) \longrightarrow M$, where $F^{-1} T N$ is the pullback bundle which has fibres $\left(F^{-1} T N\right)_{p}=T_{F(p)} N, p \in M$. Hom $\left(T M, F^{-1} T N\right)$ has a connection $\nabla$ induced from the Levi-Civita connection $\nabla^{M}$ and the pullback connection. Then the second fundamental form of $F$ is given by

$$
\left(\nabla F_{*}\right)(X, Y)=\nabla_{X}^{F} F_{*}(Y)-F_{*}\left(\nabla_{X}^{M} Y\right)
$$

for $X, Y \in \Gamma(T M)$. It is known that the second fundamental form is symmetric. First note that in [17] we showed that the second fundamental form $\left(\nabla F_{*}\right)(X, Y)$, $\forall X, Y \in \Gamma\left(\left(k e r F_{*}\right)^{\perp}\right)$, of a Riemannian map has no components in $r a n g e F_{*}$. More precisely we have the following.

Lemma 1 ([17]). Let $F$ be a Riemannian map from a Riemannian manifold $\left(M_{1}, g_{1}\right)$ to a Riemannian manifold $\left(M_{2}, g_{2}\right)$. Then

$$
g_{2}\left(\left(\nabla F_{*}\right)(X, Y), F_{*}(Z)\right)=0, \forall X, Y, Z \in \Gamma\left(\left(k e r F_{*}\right)^{\perp}\right) .
$$

Let $F$ be a Riemannian map from a Riemannian manifold $\left(M_{1}, g_{1}\right)$ to a Riemannian manifold $\left(M_{2}, g_{2}\right)$. We will use the letters $\mathscr{H}$ and $\mathcal{V}$ to denote the orthogonal projections onto distributions $\left(k e r F_{*}\right)^{\perp}$ and $k e r F_{*}$, respectively. Then we define $\mathcal{T}$ and $\mathcal{A}$ as

$$
\mathscr{A}_{E} F=\mathscr{H} \nabla_{\mathscr{H} E} \mathcal{V} F+\mathcal{V} \nabla_{\mathscr{H} E} \mathscr{H} F, \mathcal{T}_{E} F=\mathscr{H} \nabla_{\mathcal{V} E} \mathcal{V} F+\mathcal{V} \nabla_{\mathcal{V} E} \mathscr{H} F,
$$

for vector fields $E, F$ on $M_{1}$, where $\nabla$ is the Levi-Civita connection of $g_{1}$. In fact, one can see that these tensor fields are O'Neill's tensor fields which were defined for Riemannian submersions. For any $E \in \Gamma\left(T M_{1}\right), \mathcal{T}_{E}$ and $\mathcal{A}_{E}$ are skew-symmetric operators on $\left(\Gamma\left(T M_{1}\right), g\right)$ reversing the horizontal and the vertical distributions. It is also easy to see that $\mathcal{T}$ is vertical, $\mathcal{T}_{E}=\mathcal{T}_{\mathcal{V} E}$ and $\mathcal{A}$ is horizontal, $\mathcal{A}=\mathcal{A}_{\mathscr{H} E}$. We note that the tensor field $\mathcal{T}$ satisfies

$$
\mathcal{T}_{U} W=\mathcal{T}_{W} U, \forall U, W \in \Gamma\left(k e r F_{*}\right) .
$$

On the other hand, from (2.4) we have

$$
\begin{aligned}
& \nabla_{V} W=\mathcal{T}_{V} W+\hat{\nabla}_{V} W \\
& \nabla_{V} X=\mathscr{H}_{V} X+\mathcal{T}_{V} X \\
& \nabla_{X} V=\mathcal{A}_{X} V+\mathcal{V} \nabla_{X} V \\
& \nabla_{X} Y=\mathscr{H}_{X} Y+\mathcal{A}_{X} Y
\end{aligned}
$$

for $X, Y \in \Gamma\left(\left(k e r F_{*}\right)^{\perp}\right)$ and $V, W \in \Gamma\left(k e r F_{*}\right)$, where $\hat{\nabla}_{V} W=\mathcal{V} \nabla_{V} W$. In fact, these equations are O'Neill's formulas for Riemannian submersions [13]. 
From now on, for simplicity, we denote by $\nabla^{2}$ both the Levi-Civita connection of $\left(M_{2}, g_{2}\right)$ and its pullback along $F$. Then according to [12], for any vector field $X$ on $M_{1}$ and any section $V$ of $\left(\text { range } F_{*}\right)^{\perp}$, where $\left(\text { range }_{*}\right)^{\perp}$ is the subbundle of $F^{-1}\left(T M_{2}\right)$ with fiber $\left(F_{*}\left(T_{p} M\right)\right)^{\perp}$-orthogonal complement of $F_{*}\left(T_{p} M\right)$ for $g_{2}$ over $p$, we have $\nabla_{X}^{F \perp} V$ which is the orthogonal projection of $\nabla_{X}^{2} V$ on $\left(F_{*}(T M)\right)^{\perp}$. In [12], the author also showed that $\nabla^{F \perp}$ is a linear connection on $\left(F_{*}(T M)\right)^{\perp}$ such that $\nabla^{F \perp} g_{2}=0$. We now define $s_{V}$ as

$$
\nabla_{X}^{F} V=-8_{V} F_{*} X+\nabla_{X}^{F \perp} V,
$$

where $\delta_{V} F_{*} X$ is the tangential component (a vector field along $F$ ) of $\nabla_{F_{*} X}^{2} V$. It is easy to see that $\delta_{V} F_{*} X$ is bilinear in $V$ and $F_{*} X$ and $\delta_{V} F_{*} X$ at $p$ depends only on $V_{p}$ and $F_{* p} X_{p}$. By direct computations, we obtain

$$
g_{2}\left(\delta_{V} F_{*} X, F_{*} Y\right)=g_{2}\left(V,\left(\nabla F_{*}\right)(X, Y)\right),
$$

for $X, Y \in \Gamma\left(\left(k e r F_{*}\right)^{\perp}\right)$ and $V \in \Gamma\left(\left(\text { range }_{*}\right)^{\perp}\right)$. Since $\left(\nabla F_{*}\right)$ is symmetric, it follows that $\delta_{V}$ is a symmetric linear transformation of range $F_{*}$.

\section{GENERIC RIEMANNIAN MAPS FROM ALMOST HERMITIAN MANIFOLDS}

Let $F$ be a Riemannian map from an almost Hermitian manifold $\left(M, g_{M}, J\right)$ to a Riemannian manifold $\left(N, g_{N}\right)$. Define

$$
\mathscr{D}_{p}=\left(k e r F_{* p} \cap J\left(k e r F_{* p}\right)\right), p \in M
$$

the complex subspace of the vertical subspace $\mathcal{V}_{p}$

Definition 1. Let $F$ be a Riemannian map from an almost Hermitian manifold $\left(M, g_{M}, J\right)$ to a Riemannian manifold $\left(N, g_{N}\right)$. If the dimension $D_{p}$ is constant along $M$ and it defines a differentiable distribution on $M$ then we say that $F$ is a generic Riemannian map

A generic Riemannian map is purely real (respectively, complex) if $D_{p}=\{0\}$ (respectively, $D_{p}=k e r F_{* p}$ ). For a generic Riemannian map, the orthogonal complementary distribution $\mathscr{D}^{\perp}$, called purely real distribution, satisfies

$$
\operatorname{ker} F_{*}=\mathscr{D} \oplus \mathbb{D}^{\perp}
$$

and

$$
\mathscr{D} \cap \mathscr{D}^{\perp}=\{0\} .
$$

Let $F$ be a generic Riemannian map from an almost Hermitian manifold $\left(M, g_{M}, J\right)$ to a Riemannian manifold $\left(N, g_{N}\right)$. Then for $U \in \Gamma\left(k e r F_{*}\right)$, we write

$$
J U=\phi U+\omega U \text {, }
$$


where $\phi U \in \Gamma\left(k e r F_{*}\right)$ and $\omega U \in \Gamma\left(\left(k e r F_{*}\right)^{\perp}\right)$. Now we consider the complementary orthogonal distribution $\mu$ to $\omega D^{\perp}$ in $\left(k e r F_{*}\right)^{\perp}$. It is obvious that we have

$$
\phi \mathscr{D}^{\perp} \subseteq \mathscr{D}^{\perp},\left(k e r F_{*}\right)^{\perp}=\omega \mathscr{D}^{\perp} \oplus \mu .
$$

Also for $X \in \Gamma\left(\left(k e r F_{*}\right)^{\perp}\right)$, we write

$$
J X=\mathscr{B} X+\ell X,
$$

where $\mathscr{B} X \in \Gamma\left(D^{\perp}\right)$ and $\mathcal{C} X \in \Gamma(\mu)$. Then it is clear that we get

$$
\mathscr{B}\left(\left(\operatorname{ker}_{*}\right)^{\perp}\right)=\mathscr{D}^{\perp} \text {. }
$$

Considering (3.1), for $U \in \Gamma\left(k e r F_{*}\right)$, we can write

$$
J U=P_{1} U+P_{2} U+\omega U,
$$

where $P_{1}$ and $P_{2}$ are the projections from $k e r F_{*}$ to $\mathscr{D}$ and $D^{\perp}$, respectively.

We now give some examples of generic Riemannian maps from almost Hermitian manifolds to Riemannian manifolds.

Example 1. Every semi-invariant submersion [20] $F$ is a generic Riemannian map with $\left(\text { range }_{*}\right)^{\perp}=\{0\}$ and $D^{\perp}$ is a totally real distribution.

Example 2. Every generic submersion [2] $F$ is a generic Riemannian map with $\left(\text { range } F_{*}\right)^{\perp}=\{0\}$.

Example 3. Every semi-invariant Riemannian map [19] $F$ from an almost Hermitian manifold to a Riemannian manifold is a generic Riemannian map such that $D^{\perp}$ is a totally real distribution.

Example 4. Every slant- Riemannian map [21] $F$ from an almost Hermitian manifold to a Riemannian manifold is a generic Riemannian map such that $\mathscr{D}=\{0\}$ and $D^{\perp}$ is a slant distribution.

Example 5. Every semi-slant Riemannian map [15] $F$ from an almost Hermitian manifold to a Riemannian manifold is a generic Riemannian map such that $\mathscr{D}^{\perp}$ is a slant distribution.

Since semi-invariant Riemannian maps include invariant Riemannian maps and anti-invariant Riemannian maps, such Riemannian maps are also examples of generic Riemannian maps. We say that a generic Riemannian map is proper if $\mathscr{D}^{\perp}$ is neither complex nor purely real. We now present an example of proper generic Riemannian maps. In the following $\mathbb{R}^{2 m}$ denotes the Euclidean $2 m$-space with the standard metric. An almost complex structure $J$ on $\mathbb{R}^{2 m}$ is said to be compatible if $\left(\mathbb{R}^{2 m}, J\right)$ is complex analytically isometric to the complex number space $C^{m}$ with the standard flat Kählerian metric. We denote by $J$ the compatible almost complex structure on $\mathbb{R}^{2 m}$ defined by

$$
J\left(a^{1}, \ldots, a^{2 m}\right)=\left(-a^{2}, a^{1}, \ldots,-a^{2 m}, a^{2 m-1}\right) .
$$


Example 6. Consider the following map defined by

$$
F: \quad \begin{aligned}
& \mathbb{R}^{8} \\
& \left(x^{1}, x^{2}, x^{3}, x^{4}, x^{5}, x^{6}, x^{7}, x^{8}\right)
\end{aligned} \quad \longrightarrow \quad\left(x^{2}, x^{1}, \frac{x^{5}+x^{6}+x^{4}}{\sqrt{3}}, 0, \frac{x^{5}-x^{6}}{\sqrt{2}}\right) .
$$

Then we have

$$
k e r F_{*}=\operatorname{span}\left\{U_{1}=\frac{\partial}{\partial x^{8}}, U_{2}=\frac{\partial}{\partial x^{7}}, U_{3}=\frac{\partial}{\partial x^{3}}, U_{4}=-2 \frac{\partial}{\partial x^{4}}+\frac{\partial}{\partial x^{5}}+\frac{\partial}{\partial x^{6}}\right\}
$$

and

$$
\begin{aligned}
& \left(\operatorname{ker}_{*}\right)^{\perp}=\operatorname{span}\left\{Z_{1}=\frac{\partial}{\partial x^{1}}, Z_{2}=\frac{\partial}{\partial x^{2}}, Z_{3}=\frac{\partial}{\partial x^{4}}+\frac{\partial}{\partial x^{5}}+\frac{\partial}{\partial x^{6}},\right. \\
& \left.Z_{4}=\frac{\partial}{\partial x^{5}}-\frac{\partial}{\partial x^{6}}\right\} .
\end{aligned}
$$

Hence it is easy to see that

$$
g_{\mathbb{R}^{5}}\left(F_{*}\left(Z_{i}\right), F_{*}\left(Z_{i}\right)\right)=g_{\mathbb{R}^{8}}\left(Z_{i}, Z_{i}\right), i=1,2,3,4
$$

and

$$
g_{\mathbb{R}^{5}}\left(F_{*}\left(Z_{i}\right), F_{*}\left(Z_{j}\right)\right)=g_{\mathbb{R}^{8}}\left(Z_{i}, Z_{j}\right)=0,
$$

$i \neq j$, Thus $F$ is a Riemannian map. On the other hand, we have $J U_{1}=U_{2}$ and $J U_{3}=-\frac{1}{3} U_{4}+\frac{1}{3} Z_{3}$ and $J U_{4}=2 U_{3}-Z_{4}$, where $J$ is the complex structure of $\mathbb{R}^{8}$. Thus $F$ is a generic Riemannian map with $\mathscr{D}=\operatorname{span}\left\{U_{1}, U_{2}\right\}, D^{\perp}=\operatorname{span}\left\{U_{3}, U_{4}\right\}$ and $\mu=\operatorname{span}\left\{Z_{1}, Z_{2}\right\}$.

We now investigate the effect of a proper generic Riemannian map on the geometry of the total manifold, the base manifold and the map itself.

Lemma 2. Let $F$ be a proper generic Riemannian map from a Kähler manifold $\left(M, J, g_{M}\right)$ to a Riemannian manifold $\left(N, g_{N}\right)$. Then the distribution $D$ is integrable if and only if the following expression is satisfied

$$
\mathcal{T}_{X} J Y=\mathcal{T}_{Y} J X
$$

for $X, Y \in \Gamma(D)$.

Proof. From (2.2), (2.6), (3.4) and (3.6) we have

$$
\hat{\nabla}_{X} J Y+\mathcal{T}_{X} J Y=P_{1} \hat{\nabla}_{X} Y+P_{2} \hat{\nabla}_{X} Y+\omega \hat{\nabla}_{X} Y+B \mathcal{T}_{X} Y+\ell \mathcal{T}_{X} Y
$$

for $X, Y \in \Gamma(D)$. Taking the vertical parts and the horizontal parts of (3.8) we get

$$
\begin{aligned}
\hat{\nabla}_{X} J Y & =P_{1} \hat{\nabla}_{X} Y+P_{2} \hat{\nabla}_{X} Y+B \mathcal{T}_{X} Y \\
\mathcal{T}_{X} J Y & =\omega \hat{\nabla}_{X} Y+e \mathcal{T}_{X} Y
\end{aligned}
$$


Interchanging the role of $X$ and $Y$ in (3.10), and taking into account that $\mathcal{T}$ is symmetric on the vertical distribution, we derive

$$
\mathcal{T}_{X} J Y-\mathcal{T}_{Y} J X=\omega[X, Y]
$$

which gives proof.

In a similar way, we have the following lemma.

Lemma 3. Let $F$ be a proper generic Riemannian map from a Kähler manifold $\left(M, J, g_{M}\right)$ to a Riemannian manifold $\left(N, g_{N}\right)$. Then the distribution $\mathfrak{D}^{\perp}$ is integrable if and only if the following expression is satisfied

$$
\hat{\nabla}_{Z_{1}} P_{2} Z_{2}-\hat{\nabla}_{Z_{2}} P_{2} Z_{1}=P_{2}\left[Z_{1}, Z_{2}\right]+\mathcal{T}_{Z_{1}} \omega Z_{2}-\tau_{Z_{2}} \omega Z_{1}
$$

for $Z_{1}, Z_{2} \in \Gamma\left(D^{\perp}\right)$.

We now investigate the geometry of leaves of $\mathscr{D}$ and $D^{\perp}$.

Lemma 4. Let $F$ be a proper generic Riemannian map from a Kähler manifold $\left(M, J, g_{M}\right)$ to a Riemannian manifold $\left(N, g_{N}\right)$. Then the distribution $D$ defines a totally geodesic foliation in $M$ if and only if

(1) $\hat{\nabla}_{X} P_{2} Z+\mathcal{T}_{X} \omega Z$ has no components in $\mathscr{D}$ for $X \in \Gamma(\mathscr{D})$ and $Z \in \Gamma\left(\mathscr{D}^{\perp}\right)$.

(2) $\hat{\nabla}_{X} \mathscr{B} W+\mathcal{T}_{X} \in W$ has no components in $\mathscr{D}$ for $X \in \Gamma(\mathscr{D})$ and $W \in$ $\Gamma\left(\left(\operatorname{ker}_{*}\right)^{\perp}\right)$.

Proof. From (2.2), (2.6), (3.4) and (3.6) we have

$$
g_{M}\left(\nabla_{X} Y, Z\right)=-g_{M}\left(\hat{\nabla}_{X} P_{2} Z+\mathcal{T}_{X} \omega Z, J Y\right)
$$

for $X, Y \in \Gamma(\mathscr{D})$ and $Z \in \Gamma\left(D^{\perp}\right)$. This gives (1). Also from (2.2), (3.4) and (3.6) we get

$$
g_{M}\left(\nabla_{X} Y, W\right)=-g_{M}\left(\nabla_{X} \mathscr{B} W+\ell W, J Y\right)
$$

for $X, Y \in \Gamma(D)$ and $W \in \Gamma\left(\left(k e r F_{*}\right)^{\perp}\right)$. Now using (2.6) and (2.7)

$$
g_{M}\left(\nabla_{X} Y, W\right)=-g_{M}\left(\hat{\nabla}_{X} \mathcal{B} W+\mathcal{T}_{X} \lessdot W, J Y\right)
$$

which gives (2).

In a similar way, we have the following result.

Lemma 5. Let $F$ be a proper generic Riemannian map from a Kähler manifold $\left(M, J, g_{M}\right)$ to a Riemannian manifold $\left(N, g_{N}\right)$. Then the distribution $D^{\perp}$ defines a totally geodesic foliation in $M$ if and only if

(1) $\hat{\nabla}_{Z_{1}} P_{2} Z_{2}+\mathcal{T}_{Z_{1}} \omega Z_{2}=0$ for $Z_{1}, Z_{2} \in \Gamma\left(D^{\perp}\right)$.

(2) $\mathcal{C} \mathscr{H} \nabla_{Z_{1}} \omega Z_{2}+\mathcal{C} \mathcal{T}_{Z_{1}} P_{2} Z_{2}$ has no components in $\mu$.

From Lemma 4 and Lemma 5 we obtain the following decomposition theorem. 
Theorem 1. Let $F$ be a proper generic Riemannian map from a Kähler manifold $\left(M, J, g_{M}\right)$ to a Riemannian manifold $\left(N, g_{N}\right)$. Then the fibres are locally product Riemannian manifold of the form $M_{D^{D}} \times M_{D^{\perp}}$ if

(1) $\hat{\nabla}_{Y} P_{2} Z_{2}+\mathcal{T}_{Y} \omega Z_{2}=0$ for $Y \in \Gamma\left(\right.$ ker $\left._{*}\right)$ and $Z_{2} \in \Gamma\left(D^{\perp}\right)$.

(2) $\mathcal{H} \nabla_{Z_{1}} \omega Z_{2}+\mathcal{C} \mathcal{T}_{Z_{1}} P_{2} Z_{2}$ has no components in $\mu Z_{1} \in \Gamma\left(D^{\perp}\right)$.

(3) $\hat{\nabla}_{X} \mathscr{B} W+\mathcal{T}_{X} \mathcal{C W}$ has no components in $\mathscr{D}$ for $X \in \Gamma(\mathbb{D})$ and $W \in$ $\Gamma\left(\left(\operatorname{ker}_{*}\right)^{\perp}\right)$.

Lemma 6. Let $F$ be a proper generic Riemannian map from a Kähler manifold $\left(M, J, g_{M}\right)$ to a Riemannian manifold $\left(N, g_{N}\right)$. Then the distribution ker $F_{*}$ defines a totally geodesic foliation in $M$ if and only if

(1) $\hat{\nabla}_{X} P_{2} Z+\mathcal{T}_{X} \omega Z$ has no components in $\mathscr{D}$ for $X \in \Gamma(\mathscr{D})$ and $Z \in \Gamma\left(D^{\perp}\right)$.

(2) $\hat{\nabla}_{X} \mathscr{B} W+\mathcal{T}_{X} \mathcal{C W}$ has no components in $\mathscr{D}$ for $X \in \Gamma(\mathbb{D})$ and $W \in$ $\Gamma\left(\left(k e r F_{*}\right)^{\perp}\right)$.

Proof. From (2.2), (3.4) and (3.6) we have

$$
\begin{aligned}
g_{M}\left(\nabla_{U} V, \xi\right)= & g_{M}\left(\hat{\nabla}_{U} \phi V, \mathscr{B} \xi\right)+g_{M}\left(\mathcal{T}_{U} \omega V, \mathscr{B} \xi\right) \\
& +g_{M}\left(\widetilde{T}_{U} \omega V, \mathcal{C} \xi\right)+g_{M}\left(h \nabla_{U} \phi V, \mathcal{C} \xi\right)
\end{aligned}
$$

for $U, V \in \Gamma\left(k e r F_{*}\right)$ and $\xi \in \Gamma\left(\left(k e r F_{*}\right)^{\perp}\right)$. Also from (2.2), (3.4) and (3.6) we get

$$
g_{M}\left(\nabla_{X} Y, W\right)=-g_{M}\left(\nabla_{X} \mathscr{B} W+€ W, J Y\right)
$$

for $X, Y \in \Gamma(\mathscr{D})$ and $W \in \Gamma\left(\left(\operatorname{ker}_{*}\right)^{\perp}\right)$. Now using (2.6) and (2.7)

$$
g_{M}\left(\nabla_{X} Y, W\right)=-g_{M}\left(\hat{\nabla}_{X} \mathscr{B} W+\mathcal{T}_{X} \lessdot W, J Y\right)
$$

which gives (2).

In a similar way, we have the following result for distribution $\left(k e r F_{*}\right)^{\perp}$.

Lemma 7. Let $F$ be a proper generic Riemannian map from a Kähler manifold $\left(M, J, g_{M}\right)$ to a Riemannian manifold $\left(N, g_{N}\right)$. Then the distribution $\left(k e r F_{*}\right)^{\perp}$ defines a totally geodesic foliation in $M$ if and only if

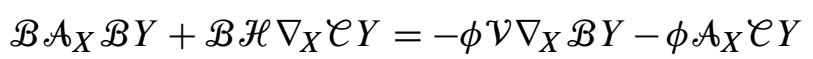

for $X, Y \in \Gamma\left(\left(k e r F_{*}\right)^{\perp}\right)$.

From Lemma 6 and Lemma 7, we have the following corollary.

Corollary 1. Let $F$ be a proper generic Riemannian map from a Kähler manifold $\left(M, J, g_{M}\right)$ to a Riemannian manifold $\left(N, g_{N}\right)$. Then $M$ is a locally product Riemannian manifold if and only if

(1) $\hat{\nabla}_{U} P_{2} Z+\mathcal{T}_{U} \omega Z$ has no components in $\mathscr{D}$ for $U \in \Gamma(\mathscr{D})$ and $Z \in \Gamma\left(D^{\perp}\right)$.

(2) $\hat{\nabla}_{U} \mathfrak{B} W+\mathcal{T}_{U} \mathcal{C W}$ has no components in $\mathscr{D}$ for $U \in \Gamma(\mathscr{D})$ and $W \in$ $\Gamma\left(\left(\operatorname{ker}_{*}\right)^{\perp}\right)$. 
(3) $\mathcal{B} \mathcal{A}_{X} \mathscr{B} Y+\mathscr{B} \mathscr{H} \nabla_{X} \mathscr{C} Y=-\phi \mathcal{V} \nabla_{X} \mathscr{B} Y-\phi \mathscr{A}_{X} \mathscr{C} Y$

for $X, Y \in \Gamma\left(\left(\text { eer }_{*}\right)^{\perp}\right)$.

In the rest of this section, we are going to find necessary and sufficient conditions for proper generic Riemannian maps from Kähler manifolds to Riemannian manifolds to be totally geodesic and harmonic, respectively. We recall that a map between Riemannian manifolds is totally geodesic if $\left(\nabla F_{*}\right)=0$ on the total manifold. A geometric interpretation of a totally geodesic map is that it maps every geodesic in the total manifold into a geodesic in the base manifold in proportion to arc lengths. We also recall that a map is harmonic if $\operatorname{trace} \nabla F_{*}=0$. Geometrically, a harmonic map $F$ is a critical point of the energy functional $E$.

Theorem 2. Let $F$ be a proper generic Riemannian map from a Kähler manifold $\left(M, J, g_{M}\right)$ to a Riemannian manifold $\left(N, g_{N}\right)$. Then $F$ is totally geodesic if and omly if the following conditions are satisfied

(1) $g_{M}\left(\mathcal{A}_{X} \phi U+\mathscr{H}_{X} \omega U, \mathscr{C} Y\right)=-g_{M}\left(\mathcal{A}_{X} \omega U+\mathcal{V} \nabla_{X} \phi U, \mathscr{B} Y\right)$,

(2) $g_{M}\left(\hat{\nabla}_{U} \mathscr{B} X+\widetilde{T}_{U} \mathcal{C} X, \phi V\right)=-g_{M}\left(\mathcal{T}_{U} \mathscr{B} X+\mathscr{H} \nabla_{U} \mathcal{C} X, \omega V\right)$,

(3) $\left(\nabla F_{*}\right)\left(X, \omega P_{2} Y+\mathcal{C} \omega Y\right)$ has no components in $\left(F_{*}(T M)\right)^{\perp}$,

for $X, Y \in \Gamma\left(\left(k e r F_{*}\right)^{\perp}\right)$ and $U, V \in \Gamma\left(k e r F_{*}\right)$.

Proof. First of all, from Lemma 1, we have $g_{M}\left(\left(\nabla F_{*}\right)(X, Y), F_{*}(Z)\right)=0$ for $X, Y, Z \in \Gamma\left(\left(k e r F_{*}\right)^{\perp}\right)$. For $X, Y \in \Gamma\left(\left(k e r F_{*}\right)^{\perp}\right)$ and $U \in \Gamma\left(k e r F_{*}\right)$, using (2.3) and (2.2), we get

$$
g_{N}\left(\left(\nabla F_{*}\right)(U, X), F_{*}(Y)\right)=g_{M}\left(\nabla_{X} J U, J Y\right) .
$$

Then from (3.4) and (3.3) we derive

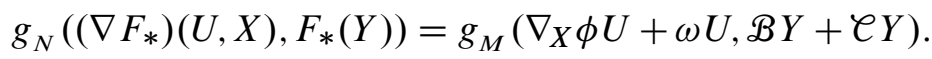

Using (2.9) and (2.8) we have

$$
\begin{aligned}
g_{N}\left(\left(\nabla F_{*}\right)(U, X), F_{*}(Y)\right)= & g_{M}\left(\mathcal{A}_{X} \phi U+\mathscr{H} \nabla_{X} \omega U, \mathscr{C Y}\right) \\
& +g_{M}\left(\mathcal{A}_{X} \omega U+\mathcal{V} \nabla_{X} \phi U, \mathfrak{B} Y\right)
\end{aligned}
$$

which gives (1). In a similar way, we obtain (2). Now, for $X, Y \in \Gamma\left(\left(k e r F_{*}\right)^{\perp}\right)$ and $\xi \in \Gamma\left(\left(F_{*}(T M)\right)^{\perp}\right)$, we have

$$
g_{N}\left(\left(\nabla F_{*}\right)(X, Y), \xi\right)=-g_{N}\left(F_{*}(Y), \nabla_{X}^{F} \xi\right) .
$$

Using (2.10) and (2.1) we get

$$
g_{N}\left(\left(\nabla F_{*}\right)(X, Y), \xi\right)=-g_{N}\left(F_{*}\left(J^{2} Y\right), \diamond_{\xi} F_{*}(X)\right) .
$$

Thus from (3.4) and (3.3), we obtain

$$
g_{N}\left(\left(\nabla F_{*}\right)(X, Y), \xi\right)=-g_{N}\left(F_{*}\left(\omega P_{2} Y\right), \diamond_{\xi} F_{*}(X)\right)-g_{N}\left(F_{*}(\mathcal{C} \omega Y), \diamond_{\xi} F_{*}(X)\right) \text {. }
$$


Now using (2.11), we arrive at

$$
g_{N}\left(\left(\nabla F_{*}\right)(X, Y), \xi\right)=-g_{N}\left(\left(\nabla F_{*}\right)\left(X, \omega P_{2} Y\right)+\left(\nabla F_{*}\right)(X, \smile \omega Y), \xi\right)
$$

which gives (3).

Theorem 3. Let $F$ be a proper generic Riemannian map from a Kähler manifold $\left(M, J, g_{M}\right)$ to a Riemannian manifold $\left(N, g_{N}\right)$. Then $F$ is harmonic if and only if

$$
\begin{aligned}
& \text { trace }\left.\right|_{\left(k e r F_{*}\right)} F_{*}\left(\mathcal{C} \mathcal{T}_{(.)} \phi(.)+\mathcal{C H} \nabla_{(.)} \omega(.)+\omega \hat{\nabla}_{(.)} \phi(.)+\omega \mathcal{T}_{(.)} \omega(.)\right)
\end{aligned}
$$

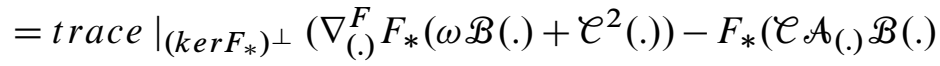

$$
\begin{aligned}
& \left.\left.+\mathcal{C H} \nabla_{(.)} \mathcal{C}(.)+\omega \mathcal{V} \nabla_{(.)} \mathscr{B}(.)+\omega \mathcal{A}_{(.)} \mathcal{C}(.)\right)\right)
\end{aligned}
$$

Proof. For $X \in \Gamma\left(\left(\mathrm{kerF}_{*}\right)^{\perp}\right)$ and $U \in \Gamma\left(\right.$ kerF $\left._{*}\right)$, from (2.2), (2.3), (3.3) and (3.4) we have

$$
\begin{aligned}
\left(\nabla F_{*}\right)(X, X)+ & \left(\nabla F_{*}\right)(U, U)=-\nabla_{X}^{F} F_{*}\left(\omega \mathscr{B} X+\ell^{2} X\right) \\
& +F_{*}\left(J\left(\nabla_{X} \mathscr{B} X+\bigodot X\right)\right)+F_{*}\left(J\left(\nabla_{U} \phi U+\omega U\right)\right)
\end{aligned}
$$

Using(3.3), (3.4) and (2.6)-(2.9) we have

$$
\begin{aligned}
& \left(\nabla F_{*}\right)(X, X)+\left(\nabla F_{*}\right)(U, U)=-\nabla_{X}^{F} F_{*}\left(\omega \mathscr{B} X+\ell^{2} X\right) \\
& +F_{*}\left(\mathcal{C} \mathcal{T}_{U} \phi U+\ell \mathscr{H} \nabla_{U} \omega(U)+\omega \hat{\nabla}_{U} \phi U+\omega \mathcal{T}_{U} \omega U\right. \\
& +F_{*}\left(\mathcal{C} \mathcal{A}_{X} \mathscr{B} X+\mathcal{C H} \nabla_{X} \mathcal{C} X+\omega \mathcal{V} \nabla_{X} \mathscr{B} X+\omega \mathcal{A}_{X} \mathcal{C} X\right)
\end{aligned}
$$

which gives our assertion.

\section{GENERIC RIEMANNIAN MAPS TO KäHLER MANIFOLDS}

In this section, we define generic Riemannian maps from Riemannian manifolds to almost hermitian manifolds, provide examples and investigate their geometric properties.

Let $F$ be a Riemannian map from a Riemannian manifold $\left(M_{1}, g_{1}\right)$ to an almost Hermitian manifold $\left(M_{2}, g_{2}, J\right)$. Define

$$
\mathfrak{D}_{p}=\left(\text { range }_{* p} \cap J\left(\text { range } F_{* p}\right)\right), p \in M_{1}
$$

the complex subspace of the image subspace $F_{*}\left(T_{p} M_{1}\right)$

Definition 2. Let $F$ be a Riemannian map from a Riemannian manifold $\left(M_{1}, g_{1}\right)$ to an almost Hermitian manifold $\left(M_{2}, g_{2}, J\right)$. If the dimension $\mathfrak{D}_{p}$ is constant along $F$ and it defines a differentiable distribution on $M_{2}$ then we say that $F$ is a generic Riemannian map.

A generic Riemannian map is purely real (respectively, complex) if $\mathfrak{D}_{p}=\{0\}$ (respectively, $\mathfrak{D}_{p}=$ range $F_{* p}$ ). For a generic Riemannian map, the orthogonal complementary distribution $\mathfrak{D}^{\perp}$, called purely real distribution, satisfies

$$
\text { range } F_{*}=\mathfrak{D} \oplus \mathfrak{D}^{\perp}
$$




$$
\mathfrak{D} \cap \mathfrak{D}^{\perp}=\{0\}
$$

Let $F$ be a generic Riemannian map from a Riemannian manifold $\left(M_{1}, g_{1}\right)$ to an almost Hermitian manifold $\left(M_{2}, g_{2}, J\right)$. Then for $F_{*}(X) \in \Gamma\left(\right.$ range $\left.F_{*}\right), X \in$ $\Gamma\left(\left(k e r F_{*}\right)^{\perp}\right)$ we write

$$
J F_{*}(X)=\varphi F_{*}(X)+\varpi F_{*}(X),
$$

where $\varphi F_{*}(X) \in \Gamma\left(\right.$ range $\left._{*}\right)$ and $\varpi F_{*}(X) \in \Gamma\left(\left(\text { range }_{*}\right)^{\perp}\right)$. Now we consider the complementary orthogonal distribution $v$ to $\varpi \mathfrak{D}^{\perp}$ in $\left(\text { range }_{*}\right)^{\perp}$. Then it is obvious that

$$
\left(\text { range } F_{*}\right)^{\perp}=\varpi \mathfrak{D}^{\perp} \oplus v, \varphi \mathfrak{D}^{\perp} \subseteq \mathfrak{D}^{\perp} .
$$

Also for $V \in \Gamma\left(\left(\text { range }_{*}\right)^{\perp}\right)$, we write

$$
J V=\mathfrak{B} V+\mathfrak{C} V,
$$

where $\mathfrak{B} V \in \Gamma\left(\mathfrak{D}^{\perp}\right)$ and $\mathfrak{C} V \in \Gamma(v)$. We also have

$$
\mathfrak{B}\left(\operatorname{range} F_{*}\right)^{\perp}=\mathfrak{D}^{\perp} .
$$

We now give some examples of generic Riemannian maps from Riemannian manifolds to almost Hermitian manifolds.

Example 7. Every CR-submanifold [4] $F$ is a generic Riemannian map with $\left(k e r F_{*}\right)=\{0\}$ and $\mathfrak{D}^{\perp}$ is a totally real distribution.

Example 8. Every generic submanifold [5] $F$ is a generic Riemannian map with $\left(\operatorname{kerF}_{*}\right)=\{0\}$.

Example 9. Every semi-invariant Riemannian map [18] $F$ from a Riemannian manifold to an almost Hermitian manifold is a generic Riemannian map such that $\mathfrak{D}^{\perp}$ is a totally real distribution.

Since semi-invariant Riemannian maps include invariant Riemannian maps and anti-invariant Riemannian maps, such Riemannian maps are also examples of generic Riemannian maps.

Example 10. Every slant Riemannian map [22] $F$ from a Riemannian manifold to an almost Hermitian manifold is a generic Riemannian map such that $\mathfrak{D}=\{0\}$ and $\mathfrak{D}^{\perp}$ is a slant distribution.

Example 11. Every semi-slant Riemannian map [16] $F$ from a Riemannian manifold to an almost Hermitian manifold is a generic Riemannian map such that $\mathfrak{D}^{\perp}$ is a slant distribution.

Example 12. Every holomorphic Riemannian map [23] is a generic Riemannian map between almost Hermitian manifolds with $\mathfrak{D}=$ range $_{*}$.

We say that a generic Riemannian map is proper if $\mathfrak{D}^{\perp}$ is neither complex nor purely real. We now present an example of a proper generic Riemannian map from a Riemannian manifold to a Kähler manifold. 
Example 13. Consider the following map defined by

$$
F: \underset{\left(x^{1}, \ldots, x^{9}\right)}{\mathbb{R}^{9}} \longrightarrow \quad \begin{gathered}
\mathbb{R}^{6} \\
\left(x^{1}, x^{9}, x^{3}, \frac{x^{4}+x^{5}}{\sqrt{2}}, \frac{x^{4}+x^{5}}{\sqrt{2}}, 0\right) .
\end{gathered}
$$

Then $k e r F_{*}$ is spanned by

$$
U_{1}=\frac{\partial}{\partial x^{2}}, U_{2}=\frac{\partial}{\partial x^{4}}-\frac{\partial}{\partial x^{5}}, U_{3}=\frac{\partial}{\partial x^{6}}, U_{4}=\frac{\partial}{\partial x^{7}}, U_{5}=\frac{\partial}{\partial x^{8}} .
$$

$\left(k e r F_{*}\right)^{\perp}$ is spanned by

$$
Z_{1}=\frac{\partial}{\partial x^{1}}, Z_{2}=\frac{\partial}{\partial x^{9}}, Z_{3}=\frac{\partial}{\partial x^{3}}, Z_{4}=\frac{1}{\sqrt{2}}\left(\frac{\partial}{\partial x^{4}}+\frac{\partial}{\partial x^{5}}\right) .
$$

Hence it is easy to see that

$$
g_{\mathbb{R}^{6}}\left(F_{*}\left(Z_{i}\right), F_{*}\left(Z_{i}\right)\right)=g_{\mathbb{R}^{9}}\left(Z_{i}, Z_{i}\right), i=1,2,3,4
$$

and

$$
g_{\mathbb{R}^{6}}\left(F_{*}\left(Z_{i}\right), F_{*}\left(Z_{j}\right)\right)=g_{\mathbb{R}^{9}}\left(Z_{i}, Z_{j}\right)=0
$$

$i \neq j$, Thus $F$ is a Riemannian map. Moreover, $\left(\text { range }_{*}\right)^{\perp}$ is spanned by

$$
V_{1}=\frac{\partial}{\partial y^{4}}-\frac{\partial}{\partial y^{5}}, V_{2}=\frac{\partial}{\partial y^{6}}
$$

On the other hand, we have $J F_{*}\left(Z_{1}\right)=F_{*}\left(Z_{2}\right)$ and $J F_{*}\left(Z_{3}\right)=\frac{1}{2} F_{*}\left(Z_{4}\right)+\frac{1}{2} V_{1}$ and $J F_{*}\left(Z_{4}\right)=-F_{*}\left(Z_{3}\right)+V_{2}$, where $J$ is the complex structure of $\mathbb{R}^{6}$. Thus $F$ is a generic Riemannian map with $\mathfrak{D}=\operatorname{span}\left\{F_{*}\left(Z_{1}\right), F_{*}\left(Z_{2}\right)\right\}, \mathfrak{D}^{\perp}=\operatorname{span}\left\{F_{*}\left(Z_{3}\right)\right.$, $\left.F_{*}\left(Z_{4}\right)\right\}$ and $v=\operatorname{span}\left\{V_{1}, V_{2}\right\}$.

We now find necessary and sufficient conditions for generic Riemannian maps from Riemannian manifolds to Kähler manifolds to be totally geodesic and harmonic. We first give the following result for totally geodesicity.

Theorem 4. Let $F$ be a proper generic Riemannian map from a Riemannian manifold $\left(M_{1}, g_{1}\right)$ to a Kähler manifold $\left(M_{2}, g_{2}, J\right)$. Then $F$ is totally geodesic if and only if

(1) for $X, Y, Y \in \in\left(\left(k e r F_{*}\right)^{\perp}\right)$,

$$
\begin{aligned}
g_{2}\left(8_{\varpi F_{*}(Y)} F_{*}(X), \mathfrak{B} V\right)= & g_{2}\left(\left(\nabla F_{*}\right)(X, Y), \mathfrak{C} V\right)+g_{1}\left(\mathscr{H} \nabla_{X} \dot{Y}^{*}{ }^{*} F_{*} \mathfrak{B} V\right) \\
& +g_{2}\left(\nabla_{X}^{F \perp} \varpi F_{*}(Y), \mathfrak{C} V\right)
\end{aligned}
$$

is satisfied, where $F_{*}(Y)=\varphi F_{*}(Y)$,

(2) $k e r F_{*}$ is totally geodesic,

(3) $\left(k e r F_{*}\right)^{\perp}$ is totally geodesic. 
Proof. For $X, Y \in \Gamma\left(\left(k e r F_{*}\right)^{\perp}\right)$ and $V \in \Gamma\left(\left(\text { range }_{*}\right)^{\perp}\right)$, using (2.2), (2.1), (4.4)and (4.3) we have

$$
g_{2}\left(\left(\nabla F_{*}\right)(X, Y), V\right)=g_{2}\left(\nabla_{X}^{F} \varphi F_{*}(Y)+\varpi F_{*}(Y), \mathfrak{B}+V \mathfrak{C} V\right) .
$$

Then from (2.10) we get

$$
\begin{aligned}
g_{2}\left(\left(\nabla F_{*}\right)(X, Y), V\right)= & g_{2}\left(\nabla_{X}^{F} F_{*}(\dot{Y}), \mathfrak{B} V+\mathfrak{C} V\right) \\
& -g_{2}\left(\delta_{\varpi F_{*}(Y)} F_{*}(X), \mathfrak{B} V\right)+g_{2}\left(\nabla_{X}^{F \perp} \varpi F_{*}(Y), \mathfrak{C} V\right) .
\end{aligned}
$$

Then (2.3) and (2.9) imply

$$
\begin{aligned}
g_{2}\left(\left(\nabla F_{*}\right)(X, Y), V\right)= & g_{2}\left(\left(\nabla F_{*}\right)(X, Y), \mathfrak{C} V\right)+g_{1}\left(\mathscr{H} \nabla_{X} Y^{*},{ }^{*} F_{*} \mathfrak{B} V\right) \\
& -g_{2}\left(\delta_{\varpi F_{*}(Y)} F_{*}(X), \mathfrak{B} V\right)+g_{2}\left(\nabla_{X}^{F \perp} \varpi F_{*}(Y), \mathfrak{C} V\right) .
\end{aligned}
$$

which gives (1). (2) and (3) can be obtained in a similar way.

For the harmonicity, we have the following result.

Theorem 5. Let $F$ be a proper generic Riemannian map from a Riemannian manifold $\left(M_{1}, g_{1}\right)$ to a Kähler manifold $\left(M_{2}, g_{2}, J\right)$. Then $F$ is a harmonic map if and only if

$$
\begin{aligned}
& \text { trace }\left.\right|_{\left(k e r F_{*}\right)^{\perp}}\left\{-\mathfrak{C}\left(\nabla F_{*}\right)\left(.,{ }^{*} F \varphi F_{*}(.)\right)-\varpi F_{*}\left(\mathscr{H} \nabla_{(.)}{ }^{*} F \varphi F_{*}(.)\right)\right. \\
& \left.-\varpi \delta_{\varpi F_{*}(.)} F_{*}(.)+e \nabla_{(.)}^{F \perp} \varpi F_{*}(.)\right\}=0
\end{aligned}
$$

and the fibers are minimal.

Proof. From (2.3), (2.2) and (4.3) we have

$$
\left(\nabla F_{*}\right)(X, X)=-J\left[\nabla_{X}^{F} \varphi F_{*}(X)+\varpi F_{*}(X)\right]-F_{*}\left(\nabla_{X}^{1} X\right)
$$

for $X \in \Gamma\left(\left(k e r F_{*}\right)^{\perp}\right)$. For $F_{*}(X)=\varphi F_{*}(X)$, from (2.3), (2.9), (2.10), (4.3) and (4.4) we get

$$
\begin{aligned}
\left(\nabla F_{*}\right)(X, X)= & -\mathfrak{B}\left(\nabla F_{*}\right)(X, \dot{X})-\mathfrak{C}\left(\nabla F_{*}\right)(X, \dot{X}) \\
& -\varphi F_{*}\left(\nabla_{X} \dot{X}\right)-\varpi F_{*}\left(\nabla_{X} \dot{X}\right)+\varphi \S_{\varpi F_{*}(X)} F_{*}(\dot{X}) \\
& +\varpi \delta_{\varpi F_{*}(X)} F_{*}(\dot{X})+\mathfrak{B} \nabla_{X}^{F \perp} \varpi F_{*}(X)+\mathfrak{C} \nabla_{X}^{F \perp} \varpi F_{*}(X) \\
& -F_{*}\left(\nabla_{X} \dot{X}\right),
\end{aligned}
$$

where $\nabla$ is the Levi-Civita connection on $M_{1}$. Now considering ( range $_{*}$ ) parts of this equation and taking trace on resulting equation we get (4.7). Second assertion comes from $\left(\nabla F_{*}\right)(U, U)=-F_{*}\left(\nabla_{U} U\right)$ for $U \in \Gamma\left(k e r F_{*}\right)$. 


\section{CONCLUSiOn REMARKS}

Generic Riemannian maps we have studied in this paper include holomorphic submanifolds, totally real submanifolds, slant submanifolds, semi-slant submanifolds, holomorphic submersions, anti-invariant submersions, semi-invariant submersions, slant submersions, semi-slant submersions, invariant Riemannian maps, antiinvariant Riemannian maps, semi-invariant Riemannian maps, slant Riemannian maps and semi-slant Riemannian maps. Thus we have constructed two wide classes of Riemannian maps. Although we give some main properties of these Riemannian maps, there are still many research problems to investigate for interested readers.

\section{REFERENCES}

[1] R. Abraham, J. Marsden, and T. Ratiu, Manifolds, Tensor Analysis, and Applications. New York: Springer, 1988.

[2] S. Ali and T. Fatima, "Generic Riemannian submersions,” Tamkang J. Math., vol. 44, pp. 395-409, 2013, doi: 10.5556/j.tkjm.44.2013.1211.

[3] P. Baird and J. C. Wood, Harmonic Morphisms Between Riemannian Manifolds. Oxford: Oxford Science Publications, Clarendon Press, 2003.

[4] A. Bejancu, Geometry of CR-submaniflods. Amsterdam: Kluwer, 1986.

[5] B. Y. Chen, "Differential geometry of real submanifolds in a Kähler manifold," Monatsh. Math., vol. 91, pp. 257-274, 1981, doi: 10.1007/BF01294767.

[6] B. Y. Chen, "Riemannian submanifolds," in Handbook of Differential Geometry, Vol. I. Amsterdam: Elsevier, 2000, pp. 187-418.

[7] M. Falcitelli, S. Ianus, and A. M. Pastore, Riemannian Submersions and Related Topics. River Edge, NJ: World Scientific, 2004.

[8] A. E. Fischer, "Riemannian maps between Riemannian manifolds," Contemp. Math., pp. 331-366, 1992.

[9] E. García-Rio and D. N. Kupeli, Semi-Riemannian Maps and Their Applications. Dordrecht: Kluwer, 1999.

[10] J. P. Jaiswal, "Harmonic maps on Sasakian manifolds,” J. Geom., vol. 104, pp. 309-315, 2013, doi: 10.1007/s00022-013-0158-2.

[11] J. P. Jaiswal and B. A. Pandey, "Non-existence harmonic maps on trans-Sasakian manifolds," Lobachevskii J.Math., vol. 37, pp. 185-192, 2016, doi: 10.1134/S1995080216020074.

[12] T. Nore, "Second fundamental form of a map," Ann. Mat. Pura Appl., vol. 146, pp. 281-310, 1987.

[13] B. O'Neill, “The fundamental equations of a submersion,” Mich. Math. J, vol. 13, pp. 458-469, 1966.

[14] B. Panday, J. P. Jaiswal, and R. H. Ojha, "Necessary and sufficient conditions for the Riemannian map to be a harmonic map on cosymplectic manifolds," Proc. Nat. Acad. Sci. India Sect. A, vol. 85, pp. 265-268, 2015, doi: 10.1007/s40010-015-0203-7.

[15] K. S. Park, "Semi-slant Riemannian maps," arXiv:1208.5362v2, 2012.

[16] K. S. Park and B. Şahin, "Semi-slant Riemannian maps into almost Hermitian manifolds," Czechoslovak Math. J., vol. 64, pp. 1045-1061, 2014, doi: 10.1007/s10587-014-0152-3.

[17] B. Șahin, "Invariant and anti-invariant Riemannian maps to Kähler manifolds," Int. J. Geom. Methods Mod. Phys., vol. 7, pp. 337-355, 2010, doi: http://dx.doi.org/10.1142/S0219887810004324.

[18] B. Șahin, "Semi-invariant Riemannian maps to Kähler manifolds," Int. J. Geom. Methods Mod. Phys., vol. 7, pp. 1439-1454, 2011, doi: http://dx.doi.org/10.1142/S0219887811005725. 
[19] B. Sahin, "Semi-invariant Riemannian maps from almost Hermitian manifolds," Indag. Math. (N.S.), vol. 23, pp. 80-94, 2012, doi: 10.1016/j.indag.2011.10.003.

[20] B. Sahin, "Semi-invariant Riemannian submersions from almost Hermitian manifolds," Canadian Mathematical Bulletin, vol. 56, pp. 173-183, 2013, doi: http://dx.doi.org/10.4153/CMB-2011144-8.

[21] B. Sahin, "Slant Riemannian maps from almost hermitian manifolds." Quaest. Math., vol. 36, pp. 449-461, 2013, doi: http://dx.doi.org/10.2989/16073606.2013.779968.

[22] B. Șahin, "Slant Riemannian maps to Kähler manifolds," Int. J. Geom. Methods Mod. Phys., vol. 10, p. 12 pp, 2013, doi: http://dx.doi.org/10.1142/S0219887812500806.

[23] B. Sahin, "Holomorphic Riemannian maps," Zh. Mat. Fiz. Anal. Geom., vol. 10, pp. 422-429, 2014.

[24] K. Yano and M. Kon, Structure on Manifolds. Singapore: World Scientific, 1984.

Author's address

B. Şahin

Inonu University, Department of Mathematics, 44280, Malatya, Turkey

E-mail address: bayram. sahin@ymail.com

Current address: Ege University, Department of Mathematics, 35100, Izmir, Turkey

E-mail address: bayram. sahinege.edu.tr 